\title{
Real-Time Environmental Parameters Monitoring System
}

\author{
Davor Ćorić \\ Faculty for Information systems and Technologies, University of Donja Gorica
}

\section{Abstract}

The objective of the RTEPMS (Real-Time Environmental Parameters Monitoring System) project is to design and implement a general-purpose environmental parameter monitoring system. The main focus is on measuring water quality parameters is several use cases. To achieve this goal, monitoring will be based on widely available, relatively cheap Internet of Things (IOT) based hardware. One of the most important use cases, drinking water quality monitoring, is piloted in cooperation with Regional Waterworks for the Montenegrin Cost (RWMC). RWMC is the largest drinking water supply company in Montenegro. Using the $135 \mathrm{~km}$ of pipelines it delivers drinking water of the highest quality to the Montenegrin coast. Beside drinking water monitoring use case, other use cases will be implemented and executed, such as water quality monitoring for fish farming as well as the pool (swimming) water quality monitoring.

Keywords: platform, data integration, smart system, processing, sensor JEL classification: Z18, Q59

Acknowledgments: This project is supported by the Ministry of Science of Montenegro under contract number 01-2312/4.

\section{Introduction}

Water is essential for human life. By 2015. almost $90 \%$ of the population had access to a water from a source that is suitable for drinking (World Health Organization, 2019). Drinking water affects our health and that is why water quality monitoring is of a great importance. Water quality highly depends on the way of its exploitation, world's reserves of drinking water are in constant decrease due to consumption increase, constant pollution and poor management of water resources (World Health Organization, N/A). Since drinking water is one of the most significant resources in the Montenegro, there is a lot of place for improvement in water resource management.

\section{Context}

The heart of the RWMC water supply system is underground spring "Bolje sestre" located near the Skadar Lake. Water from this spring belongs to Al category meaning that it can be bottled or put into water supply system just after the disinfection process. Current quality monitoring routine considers periodical physical-technical and microbiotical measurements manually done by the employee and controlled by national laboratories. Due to very specific position of the spring it is not rare situation that spring water quality gets influenced by external factors especially bad weather conditions (heavy rain). In order to be able to react on time, there is obvious need for real-time water quality monitoring system. 
The main objective of the RTEPMS project is to implement, test and validate general purpose platform for environmental parameter tracking with focus on water quality assessment. The project implementation is divided into two phases:

- cloud-based Data Integration Platform development alongside with sensor nodes,

- development of expert modules for various water quality use cases (drinking water monitoring, fish farm water monitoring, swimming water monitoring, etc.).

\section{Implementation}

The main idea is to develop easily extensible platform for general-purpose measurement tracking. With the ability to expose main platform interfaces for data collection or data retrieval, integration with third-party applications will be possible. Sensor nodes will be designed to support water quality measurement scenarios. Based on cheap, broadly available lora (Espressif Systems, 2019) sensors for water quality it is expected to achieve significantly lower price for single sensor node than it is possible with current commercial solutions.

The platform implementation will be based on open standards and one of the goals it to achieve high interoperability with existing solutions. Overview of the RTEPMS system architecture is depicted in Figure 1.

Figure 1

RTEPMS System Architecture View

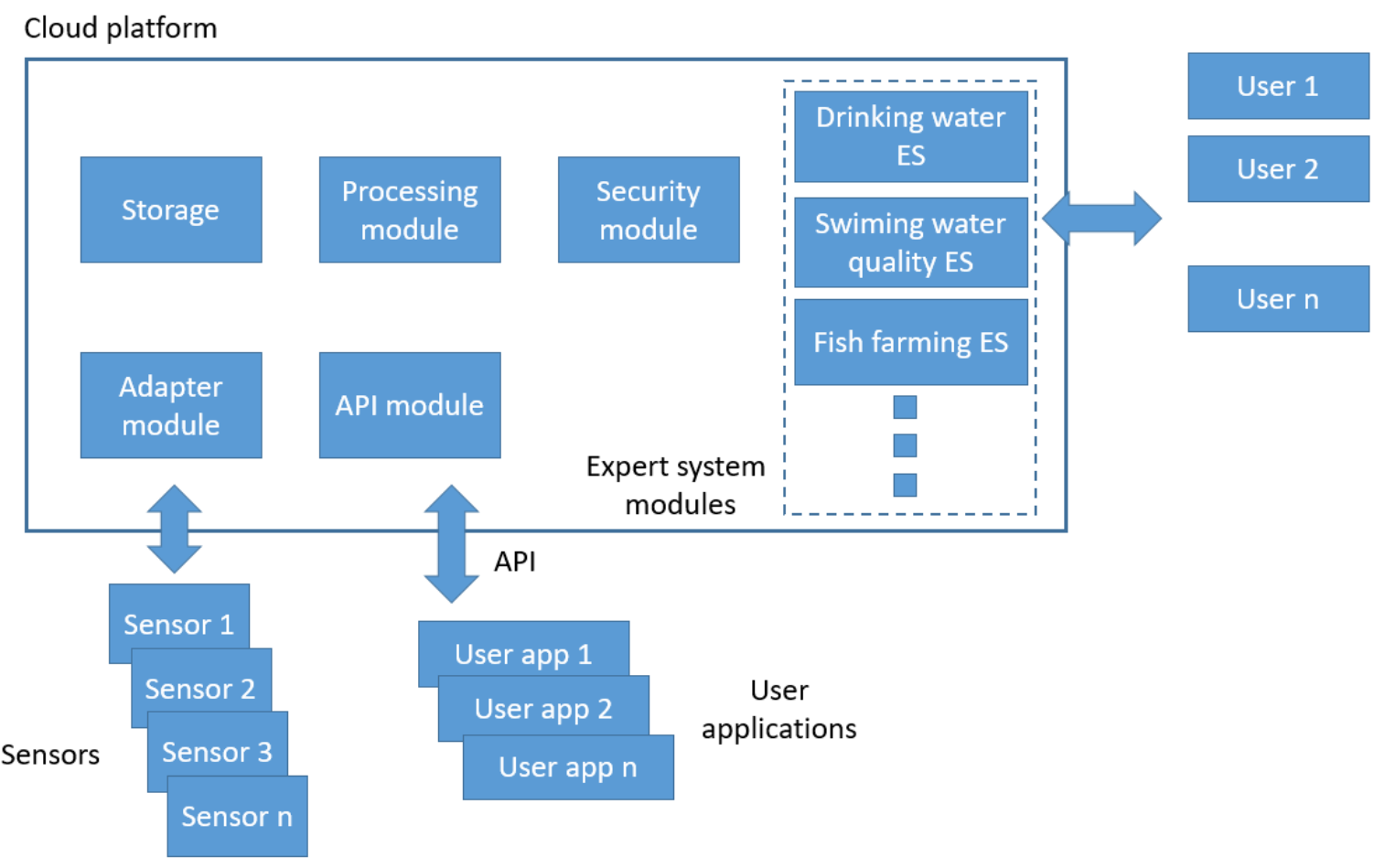

Source: Authors' illustration

As shown in Figure 1. the platform will serve as exchange point between sensor nodes, sending the measurements and the end user applications. Expert system modules will offer enriched data analytic features with alerting abilities in order to notify platform users in case that something is out of order. 
First phase of the implementation will be focused on the Data Integration Platform (DIP) development and sensor node development. Sensor nodes should be capable of delivering measurements to the data integration platform through several types of communication channels (WiFi, LoRa, 3G) (Sinha et al., 2017). DIP will be cloud hosted application with ability to receive, serve and visualize collected data.

Second phase of the implementation will focus on development of the expert modules. The main task is to develop advisory oriented models based on the collected data and specific domain expertise. It is expected to develop at least three expert modules in that manner.

\section{Data Integration Platform}

Data Integration Platform represents the core component for the real-time environmental parameter monitoring system. While hosted in the cloud DIP will handle crucial following tasks:

- collecting (receiving) data sent from sensor nodes,

- processing, validating and aggregating received data,

- permanently storing data,

- providing easy and intuitive way for data extraction.

Beside handling the sensor data DIP will have embedded expert module system which will provide for end users better insight into collected data. It is important to note that each expert module will have implemented independent heuristics for specific piloting scenario. Expert modules will be able to trigger alarms and certain actions based on the collected data. Implementation view of the DIP is shown in Figure 2.

Figure 2

Implementation View of the Data Integration Platform

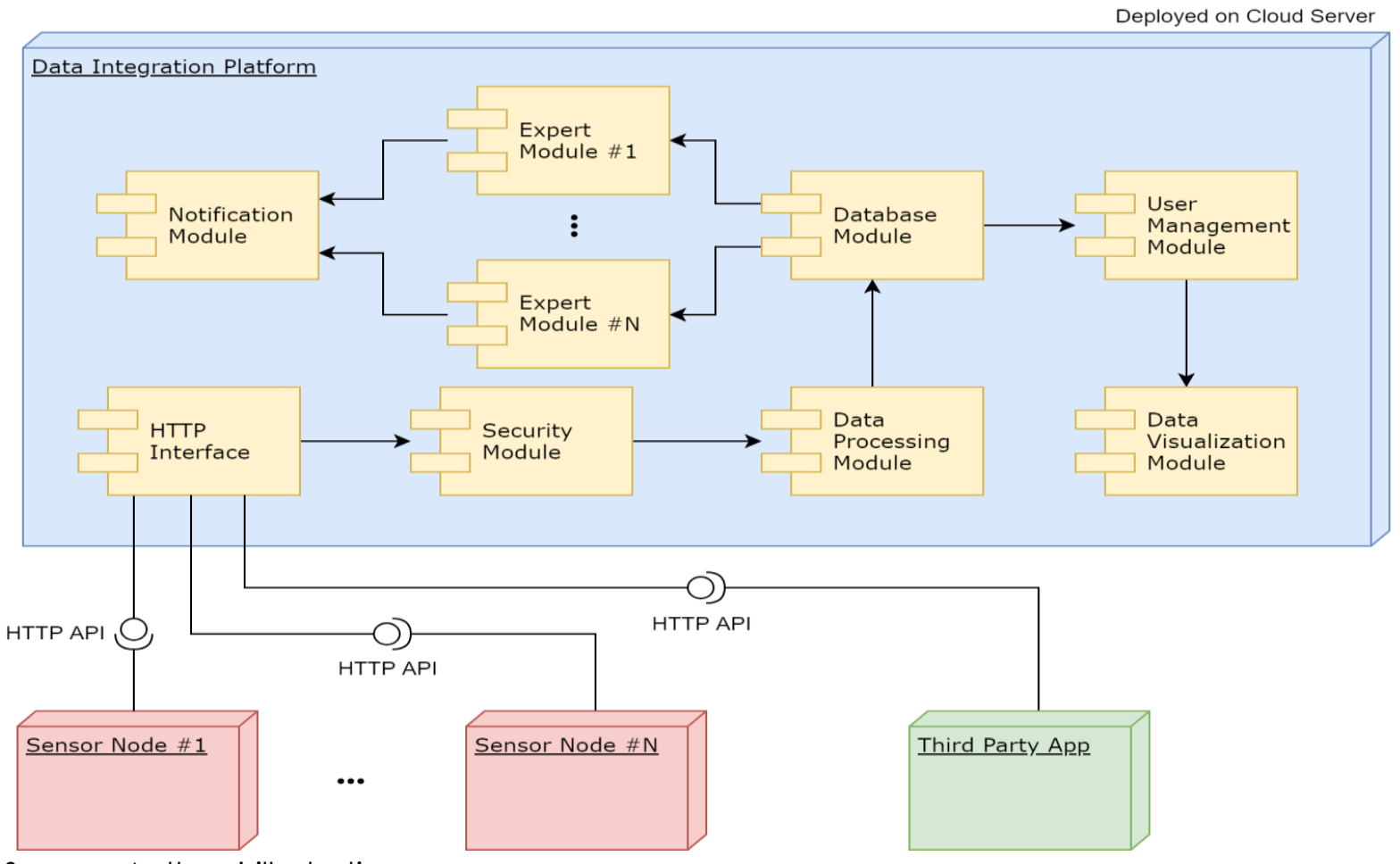

Source: Authors' illustration 
Figure 2. depicts internal organization of the DIP components as well as the possibility to build external third-party application utilizing core DIP functionalities.

Data Integration Platform will be deployed on the cloud server with ability to scale based on the server load. This will ensure easy vertical scaling if needed, however due to nature of environmental parameters and their speed of change load issues are not expected.

Main communication protocol should be HTTP which is well supported by sensor nodes and back-end services. Therefore, continuous Internet access is anticipated on the pilot test sites in order to keep measurements coming. If reliable Internet connection turns out to be an issue on the piloting sites certain measurement caching techniques are considered in order to save the measured data until the Internet connection becomes available.

Security of the platform will rely on the standard practices in terms of user authentication and authorization, restricted access and light encryption suitable for battery powered loT devices.

Data exchange between various platform components will utilize JSON format (Bray, 2017). Since components are designed as stand-alone systems, micro-serviceoriented backend will be developed as a perfect fit. This approach will reduce mutual component dependency and loose coupling architecture will be achieved.

\section{Sensor Nodes}

Sensor nodes represent modular hardware components of the RTEPMS system. Sensor node main task is to get measured values from the sensors and send them to the Data Integration Platform. Each sensor node should be capable of measuring multiple parameters of interest, pre-process them and send measurements via HTTP to the DIP. Architecture of a single modular sensor node is given in Figure 3.

Figure 3

Sensor Node Architecture

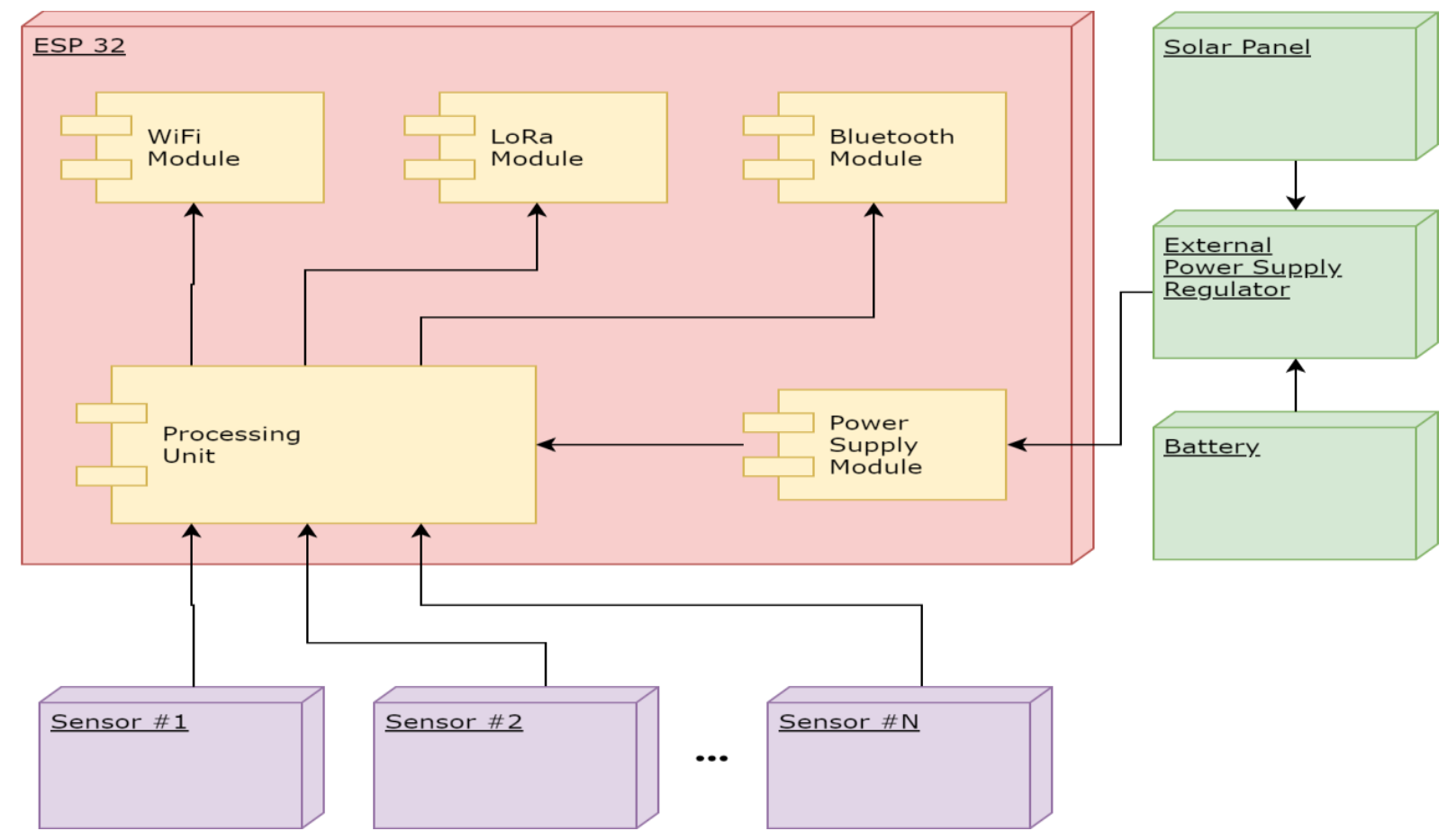

Source: Authors' illustration 
For hardware stack ESP32 board is chosen. ESP32 is low-cost, low-powered, systemon-a-chip microcontroller. ESP32 offers integrated WiFi, LoRa and Blvetooth connectivity (Gubbi et al., 2013). The main idea is to use WiFi where it is possible and directly communicate with the DIP over HTTP requests. However, if that is not possible there is possibility to utilize integrated LoRa or Bluetooth modules in order to pass the measured values to the gateway. Gateway must have Internet access in order to pass received data to the DIP as DIP communicates only through HTTP requests.

Single sensor node power consumption highly depends on the connected sensors. The ESP32 itself supports several power-saving modes, however by design it is envisaged to implement solar-based power supply [6json]. It is expected that two-day only battery autonomy is sufficient to keep the sensor node running through the year without interruptions. Nevertheless, it is expected that battery powered sensor node (with 12V 9Ah batteries) should keep the sensor node running for at least 45 days under the assumption that measurements are sent every 15 minutes, and that sensor reading process takes up to 90 seconds powered by 40mA. In idle state, ESP32 should be in "deep sleep" mode and consume not more than $3 \mathrm{~mA}$.

Although RTEPMS is designed to be general-purpose parameter monitoring platform, for this project several sensors listed in Table 1. will be used in pilot sites in order to support test scenarios.

Table 1

List of Available Sensors for Pilot Scenarios

\begin{tabular}{cl}
\hline$\#$ & \\
\hline 1. & Waterproof temperature sensor. \\
\hline 2. & Analog conductivity sensor $(\mathrm{K}=0.1-\mathrm{K}=1)$. \\
\hline 3. & Analog $\mathrm{PH}$ sensor $(0-14 \mathrm{pH})$ \\
\hline 4. & Analog Oxide-Reduction Potential sensor. \\
\hline 5. & Analog Total Dissolved Solids sensor. \\
\hline 6. & Analog Turbidity sensor. \\
\hline 7. & Analog Dissolved Oxygen sensor. \\
8. & Non-contact liquid level sensor. \\
10. & Analog Water Pressure sensor \\
11. & Air humidity sensor. \\
12. & Wind direction sensor (wind vane). \\
13. & Wind speed sensor (anemometer). \\
14. & Atmosphere pressure sensor. \\
\hline
\end{tabular}

Source: Authors' work

\section{Conclusion}

The RTEPMS platform will provide complete solution for data collection, processing and decision making in an efficient way. With focus on water quality expert modules platform will offer important insight into ongoing processes and make data-driven decisions.

Open architecture makes the platform hardware agnostic in terms of selected sensors and other equipment. On top of that, publicly exposed API will open opportunities for third party application or expert module development.

At the final stage, platform should have potential for commercialization through the Institute for Water Resources which will be founded during the lifetime of this project. 


\section{References}

1. Bray, T. (2017), "The javascript object notation (json) data interchange format", No. RFC 8259.

2. Espressif Systems (2019), "ESP32 Series Datasheet", available at: https://www.espressif.com/sites/default/files/documentation/esp32_datasheet_en.pd f (25 April 2019).

3. Gubbi, J., Buyya, R., Marusic, S., Palaniswami, M. (2013), "Internet of Things (loT): A vision, architectural elements, and future directions", Future generation computer systems, Vol. 29, No. 7, pp. 1645-1660.

4. Sinha, R. S., Wei, Y., Hwang, S. H. (2017), "A survey on LPWA technology: LoRa and NBIOT", Ict Express, Vol. 3, No. 1, pp. 14-21.

5. World Health Organization (2019), "Drinking Water Facts", available at: https://www.who.int/en/news-room/fact-sheets/detail/drinking-water (25 April 2019).

6. World Health Organization (N/A), "Drinking Water", available at: https://www.who.int/water sanitation health/monitoring/water.pdf (25 April 2019).

\section{About the author}

Davor Ćorić, MsC is a teaching assistant at University Donja Gorica Montenegro.He received Msc in Internatonal economy an electrical scienece. His main research interests are ICT, Project management and digitalization of economics processes. The author can be contacted at davor.coric@udg.edu.me. 